\title{
Distinct cognitive profiles and rates of decline on the Mattis Dementia Rating Scale in autopsy-confirmed frontotemporal dementia and Alzheimer's disease
}

\author{
KATYA RASCOVSKY, ${ }^{1}$ DAVID P. SALMON, ${ }^{2}$ LAWRENCE A. HANSEN, ${ }^{2,3}$ \\ AND DOUGLAS GALASKO ${ }^{2,4}$ \\ ${ }^{1}$ Memory and Aging Center, University of California, San Francisco, California \\ ${ }^{2}$ Department of Neurosciences, University of California, San Diego, California \\ ${ }^{3}$ Department of Pathology, University of California, San Diego, California \\ ${ }^{4}$ Neurology Service, San Diego Veteran Affairs Medical Center, La Jolla, California \\ (Received October 23, 2007; Final Revision December 27, 2007; Accepted December 28, 2007)
}

\begin{abstract}
Neuropsychological studies have shown that patients with Frontotemporal dementia (FTD) perform worse than patients with Alzheimer's disease (AD) on tests of conceptualization and verbal fluency, but better on tests of memory and visuospatial functions. However, it is not known if these distinct cognitive profiles are robust enough to be detected using a relatively brief dementia screening instrument such as the Mattis Dementia Rating Scale (MDRS). To address this issue, the MDRS subscale profiles of patients with autopsy-confirmed FTD $(n=17)$ or AD $(n=34)$ were compared. Results showed distinct cognitive profiles in which FTD patients performed worse than AD patients on the Initiation/Perseveration and Conceptualization subscales while performing better on the Memory and Construction subscales. The distinct subscale profiles correctly classified $85 \%$ of AD patients and $76 \%$ of FTD patients. Profiles were maintained in a subset of mildly-to-moderately demented patients (MDRS $\geq 105$ ) and correctly classified $89 \%$ of these patients. In addition, FTD patients (mean $=30.0$ points/year) declined faster than $\mathrm{AD}$ patients (mean $=14.8$ points/year) on MDRS total and specific subscale scores. These results suggest that the MDRS may be a useful adjunct to other clinical measures for distinguishing FTD from AD and tracking the progression of the disorder. (JINS, 2008, 14, 373-383.)
\end{abstract}

Keywords: Frontotemporal dementia, Alzheimer's disease, Mattis Dementia Rating Scale, Rate of progression, Cognitive profile, Functional impairment

\section{INTRODUCTION}

Frontotemporal dementia (FTD) is the overarching label used to describe a spectrum of neurodegenerative disorders characterized by relatively circumscribed frontal and temporal lobar atrophy (Kertesz et al., 2003b). In the usual case, FTD begins insidiously with personality and behavioral changes such as inappropriate social conduct, apathy, disinhibition, perseverative behavior, loss of insight, and hyperorality. These changes are invariably accompanied by (or soon followed by) cognitive deficits, including alter-

Correspondence and reprint requests to: David P. Salmon, Ph.D., Alzheimer's Disease Research Center, University of California, San Diego, 9500 Gilman Drive, La Jolla, CA 92093-0948. E-mail: dsalmon@ucsd.edu ations in so-called frontal "executive" functions and/or aphasia, often with relative sparing of visuospatial abilities and memory (Neary et al., 1998; Rosen et al., 2002, 2004). FTD is pathologically heterogeneous and includes taupositive pathology with or without Pick bodies (i.e., Pick's disease); tau-negative, ubiquitin-positive inclusions associated with motor neuron disease (FTD-MND); or may lack distinctive histopathology (i.e., dementia lacking distinctive histopathology; DLDH; McKhann et al., 2001). It is estimated that approximately 3-20\% of all cases of dementia may be FTD (Barker et al., 2002; Gislason et al., 2003; Knopman et al., 1990; Neary, 1999; Ratnavalli et al., 2002; Rosso et al., 2003), and the disorder is particularly prevalent when the age of onset of dementia is younger than 65 years (Ratnavalli et al., 2002; Rosso et al., 2003). Recent 
studies suggest that FTD follows a particularly malignant course (Grasbeck et al., 2003; Hodges et al., 2003), but information as to the nature and rate of cognitive decline is still scarce (Binetti et al., 2000; Pasquier et al., 2004; Rascovsky et al., 2005).

Although histopathologically distinct from the more common Alzheimer's disease (AD), FTD is often difficult to differentiate from probable AD during life (Litvan et al., 1997; Mendez et al., 1993; Varma et al., 1999). Accurate differential diagnosis is crucial, however, given potential differences in prognosis (Rascovsky et al., 2005) and in pharmacological management strategies that might be suggested by different neurotransmitter system involvement in the two disorders (Lebert et al., 2004; Moretti et al., 2003; Pasquier et al., 2003; Qume et al., 1994; Rahman et al., 1999; Sparks \& Markesbery, 1991; Swartz et al., 1997). Recent studies using batteries of neuropsychological tests suggest that FTD and AD are associated with distinct cognitive profiles that might aid in their differential diagnosis (Binetti et al., 2000; Elfgren et al., 1994; Frisoni et al., 1995; Gregory et al., 1997; Kramer et al., 2003; Lindau et al., 1998; Mendez et al., 1996; Pachana et al., 1996; Rascovsky et al., 2002; Thomas-Anterion et al., 2000). Particularly compelling are retrospective studies that have shown a double dissociation in which mildly-to-moderately demented patients with autopsy-confirmed FTD are more impaired than those with autopsy-confirmed AD on tests sensitive to frontal lobe dysfunction (e.g., word generation tasks), but less impaired on tests of memory and visuospatial abilities sensitive to dysfunction of medial temporal and parietal association cortices (e.g., Rascovsky et al., 2002). In some cases, these distinct cognitive profiles are able to differentiate between patients with autopsy confirmed FTD or AD with greater than $85 \%$ accuracy (Rascovsky et al., 2002).

Given that different cognitive deficit profiles are exhibited by patients with FTD and AD on neuropsychological test batteries, it is possible that these differences may be robust enough to be detected with relatively brief dementiascreening instruments that tap multiple cognitive functions (Bier et al., 2004; Kertesz et al., 2003a; Mathuranath et al., 2000; Slachevsky et al., 2004). An instrument that may be particularly effective in this regard is the Mattis Dementia Rating Scale (MDRS; Mattis, 1976, 1988). The MDRS is a widely used standardized mental status examination that provides a global measure of dementia derived from subscales for five cognitive capacities: attention, initiation and perseveration, construction, conceptualization, and memory. It is a reliable (Coblentz et al., 1973; Gardner et al., 1981; Smith et al., 1994; Vitaliano et al., 1984) and valid psychometric instrument for detecting and staging dementia (Gardner et al., 1981; Green et al., 1995; Monsch et al., 1995; Salmon et al., 1990; Shay et al., 1991; Smith et al., 1994; Troster et al., 1994; Woodard et al., 1996). Several studies have shown that the pattern of MDRS subscale scores can distinguish between $\mathrm{AD}$ and a variety of other dementing illnesses such as dementia with Lewy Bodies (DLB;
Aarsland et al., 2003; Connor et al., 1998), Parkinson's disease (Paolo et al., 1995), Huntington's disease (Paulsen et al., 1995; Rosser \& Hodges, 1994; Salmon et al., 1989), and progressive supranuclear palsy (Rosser \& Hodges, 1994). The MDRS is also effective for tracking cognitive decline over time (Galasko et al., 2000; Gould et al., 2001; Salmon et al., 1990) and might have the capacity to detect differences between FTD and AD in the rate of global cognitive decline (cf., Rascovsky et al., 2005) and in the rate of decline in specific cognitive abilities assessed by the MDRS subscales. Accordingly, the present study was designed to determine whether autopsy-confirmed FTD and AD patients exhibit distinct patterns of cognitive deficits on the MDRS, and to compare the rate and nature of decline on the MDRS in the two groups over a 12-month period.

\section{METHODS}

\section{Subjects}

A total of 17 patients with autopsy-confirmed FTD and 34 patients with autopsy-confirmed AD were included in the present study. All patients had been participants at the University of California, San Diego (UCSD) Alzheimer's Disease Research Center (ADRC), through which they received annual medical, neurological, and neuropsychological evaluations. The FTD patients represented a series of consecutive cases that had exhibited progressive dementia, completed neuropsychological testing, and received a neuropathological diagnosis of FTD at autopsy. FTD patients were included in the present study based only on pathologic criteria (described below), regardless of the initial clinical presentation. The initial clinical diagnoses of the FTD patients during life were varied and included probable $\mathrm{AD}(n=6)$, possible $\mathrm{AD}(n=5)$, Pick's disease $(n=3)$, amyotrophic lateral sclerosis (ALS) dementia $(n=2)$, and possible multiinfarct dementia $(n=1)$. It should be noted, however, that many of these subjects were clinically diagnosed before the development and application of standardized clinical criteria for FTD (e.g., Neary et al., 1998). None of the FTD patients were clinically diagnosed with a language variant of FTD (e.g., Semantic Dementia, progressive nonfluent aphasia), although four had prominent language deficits in addition to typical behavioral changes of FTD.

The AD patients were randomly selected from a larger series of autopsy-confirmed cases that had exhibited progressive dementia and completed neuropsychological testing, with the restriction that two AD patients matched each FTD patient in terms of age at time of testing, years of education, and overall level of dementia as evidenced by total score on the MDRS. Patients were matched within \pm 5 points on total MDRS scores. As expected from the matching procedure, the groups did not differ significantly in age at time of testing $[t(49)=1.72 ; p>.05]$, years of education $[t(49)<1]$, total MDRS scores $[t(49)<1]$, or estimated duration of symptoms before their initial ADRC assessment $[t(49)<1]$. They also did not differ in level of 
disability in instrumental activities as measured by the Functional Activities Questionnaire (FAQ) $[t(47)<1]$ or gender distribution [FTD: males $=12$, females $=5 ;$ AD: males $=24$, females $\left.=10 ; \chi^{2}(1,51)=0.0 ; p>.05\right]($ see Table 1$)$. The initial clinical diagnoses of the AD patients included probable $\mathrm{AD}(n=25)$, possible $\mathrm{AD}(n=7)$, and questionable dementia $(n=2)$. The patients with questionable dementia had cognitive changes that were not sufficient to significantly interfere with activities of daily living, and all of them eventually received a diagnosis of probable or possible AD as they were followed longitudinally at the UCSD ADRC.

A subgroup of 12 FTD and 24 AD patients received an additional evaluation approximately 1 year after the first. The demographic characteristics of this subgroup of patients did not differ from those of the total sample and the groups remained matched on age at time of baseline testing [FTD = 64.7 years; $\mathrm{AD}=66.8$ years; $t(34)=1.03 ; p>.05$ ], years of education $[\mathrm{FTD}=13.67$ years; $\mathrm{AD}=14.04$ years; $t(34)<1]$, total MDRS scores at year 1 [FTD $=103.1$, $\mathrm{AD}=102.9 ; t(34)<1]$, and FAQ score at year 1 [FTD = $66.2, \mathrm{AD}=66.8 ; t(33)<1]$. The groups were also matched in time elapsed between baseline (year 1) and follow-up (year 2 ) testing $[\mathrm{FTD}=1.1$ years, $\mathrm{AD}=1.1$ years; $t(34)<1]$. Three AD patients were treated with cholinergic medications during the study interval; no FTD patients received cholinergic drugs during this period.

\section{Neuropathology}

Autopsy was performed according to established UCSD ADRC protocols (Hansen \& Samuel, 1997). Briefly, the left hemibrain was fixed in $10 \%$ formalin for 5-7 days from which paraffin-embedded sections were made and stained with hematoxylin and eosin (H\&E), thioflavin-S, and antibodies against ubiquitin and a phosphorylated form of tau (AT8; McKhann et al., 2001). Total plaque, neuritic plaque, and neurofibrillary tangle counts were then performed on midfrontal, rostral superior temporal, inferior parietal, entorhinal, and hippocampal regions. Brains were then staged for the degree of neurofibrillary pathology by a modification (Samuel et al., 1996) of the method of Braak and Braak (1991) by one neuropathologist (L.A.H.). Clinicopathological diagnosis of AD was made according to both National Institutes of Aging (NIA; Khachaturian, 1985) and the Consortium to Establish a Registry for Alzheimer's Disease (CERAD) criteria (Mirra et al., 1991). None of the AD patients had a known genetic variant of AD or a strong positive family history of the disease. Among FTD cases, Pick's disease was defined by the presence of Pick bodies in the hippocampal granule cell layer and/or pyramidal cell layer of the frontal and temporal cortices. Pick bodies were identified by their characteristic morphology on H\&E sections. Cases with tau-negative, ubiquitin-positive inclusions in brainstem motor nuclei and/or hippocampus (typical of motor neuron disease, MND) were classified as FTD with MND-type inclusions (although none of these subjects received a clinical diagnosis of ALS during life). Finally, cases with nonspecific atrophy and spongy vacuolization in frontal and/or temporal areas in the absence of silver, tau, or ubiquitin-positive intraneuronal inclusions were defined as dementia lacking distinctive histology (DLDH). All FTD brains lacked significant AD pathology. In the present sample, three FTD patients had Pick's disease strictly defined by the presence of Pick bodies, six had ubiquitin-positive inclusions of the MND-type (McKhann et al., 2001), two had a clinicopathologic diagnosis of FTD-MND, one had tauopathy with a distribution similar to that of corticobasal degeneration (CBD), and the remaining five were classified as DLDH. None of the FTD patients had a known genetic variant of FTD or a strong positive family history of the disease. In the subgroup of 12 FTD patients with longitudinal evaluations, 2 had Pick's disease strictly defined by

Table 1. Mean age (in years), years of education, Mattis Dementia Rating Scale (MDRS) total scores, Functional Activities Questionnaire (FAQ) percentage scores, and estimated duration of symptoms (in years) of patients with Frontotemporal Dementia (FTD) or Alzheimer's Disease (AD) at time of testing

\begin{tabular}{lccc}
\hline \hline & FTD & AD & \\
& $N=17$ & $N=34$ & $p$ value \\
\hline Age $(S D)$ & $63.00(7.9)$ & $66.09(4.9)$ & .092 \\
Range & $(48-76)$ & $(57-76)$ & .407 \\
Education $(S D)$ & $13.24(4.2)$ & $14.06(2.8)$ & \\
Range & $(3-19)$ & $(8-19)$ & .979 \\
MDRS score $(S D)$ & $103.29(26.6)$ & $103.09(26.3)$ & .738 \\
Range & $(35-139)$ & $(30-139)$ & .722 \\
FAQ \% score $(S D)$ & $63.34(25.3)^{\mathrm{a}}$ & $(11-100)$ & \\
Range & $(12-95)$ & $4.62(2.7)$ & $(2-15)$ \\
Duration of symptoms $(S D)$ & $4.29(3.6)$ & $(1-16)$ & \\
Range &
\end{tabular}

$\mathrm{a}_{n}=16$.

$\mathrm{b}_{n}=33$. 
the presence of Pick bodies, 3 had ubiquitin-positive inclusions of the MND-type, 1 had a clinicopathologic diagnosis of FTD-MND, 1 had tauopathy with a distribution similar to that of $\mathrm{CBD}$, and the remaining 5 were classified as DLDH.

\section{Procedures}

The MDRS was administered to each patient according to published procedures (Mattis, 1988), with the exception that all items were administered to each patient. Patients were tested individually by a trained psychometrist in a quiet, well-illuminated room. The MDRS is a standardized, 144-point mental status examination consisting of five subscales that assess Attention (37 points), Initiation/ Perseveration (37 points), Construction (6 points), Conceptualization (39 points), and Memory (25 points). A knowledgeable informant was also interviewed to complete the FAQ (Pfeffer et al., 1982), a measure of the patient's ability to perform instrumental activities of daily living (e.g., keep appointments, prepare meals). The FAQ was scored in terms of the percent of possible points that were obtained based only on those items that the patient had engaged in before the development of dementia. Informed consent was obtained from the patient and caregiver (usually the next of kin) before neuropsychological testing and after the procedures of the study had been fully explained. The study procedures conformed to Federal guidelines for the protection of human subjects and were reviewed and approved by the UCSD Institutional Review Board.

\section{Data Analysis}

The MDRS subscales contain different numbers of points, so individual scores were converted into percentage of maximum possible score to put all subscales on the same metric before profile analysis. Because patients in the two groups were matched for total DRS score, it is not necessary to normalize scores to determine whether the various subscales (i.e., cognitive processes) differentially contribute to total DRS score (i.e., global level of dementia) in the FTD and AD groups. Profile analysis was performed using repeated measures multivariate analysis of variance (RM MANOVA). Backward Stepwise Logistic Regression analysis was used to identify the subscale or combination of subscales that best differentiated between FTD and AD patients. Repeated measures analysis of variance (RM ANOVA) was used to explore rate of change on the MDRS. Annualized rates of change (ARC) in MDRS total and subscale scores for each patient were calculated using the following formula:

$\mathrm{ARC}=\left[\frac{\text { score at evaluation } 2-\text { score at evaluation } 1}{\text { months between evaluation }}\right] \times 12$.

Independent sample $t$ tests were used to compare ARC between groups. Effect sizes were calculated using Cohen's d. Given the modest sample sizes and the relatively few comparisons, $\alpha$ was set at .05 (two-tailed) and no correction for Type I error was used. All statistical analyses were performed using SPSS version 11.0 (SPSS; Chicago, IL).

\section{RESULTS}

\section{Cognitive Profiles at Baseline}

A profile analysis using RM MANOVA revealed a significant subscale $\times$ group interaction [multivariate $F(4,46)=$ 9.95; $p<.001]$ reflecting distinct cognitive profiles for each group (see Figure 1). FTD patients scored lower than

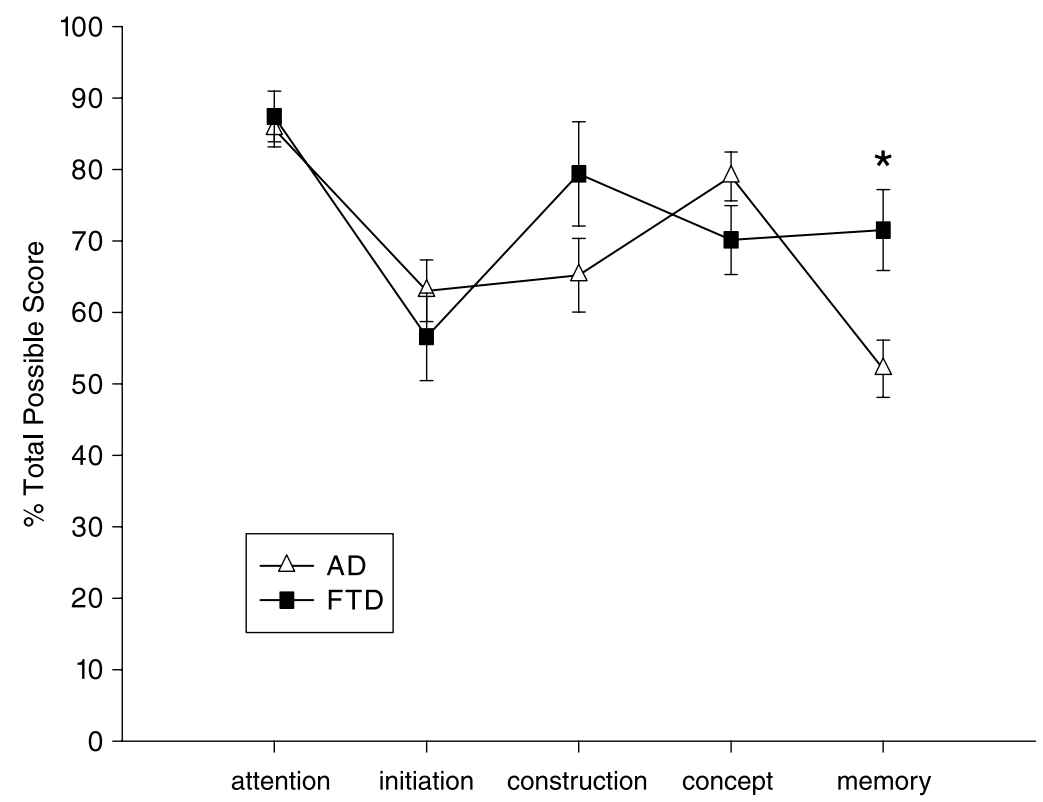

Fig. 1. Mean percentage (\%) of total possible score achieved by patients with FTD (squares) or AD (triangles) on the Mattis Dementia Rating Scale (MDRS) subscales. Error bars denote standard error of the mean. *Significant group difference, $p<.05$. 
AD patients on the Initiation/Perseveration and Conceptualization subscales, but higher on the Construction and Memory subscales. Despite a significant overall group profile difference, the only individual difference that was statistically significant was on the Memory subscale $[F(1,49)=$ 7.78; $p<.05 ; \mathrm{d}=0.83$ ]. A logistic regression model that included the Initiation/Perseveration, Construction, Conceptualization, and Memory subscales was significant $\left[\chi^{2}(1,51)=33.73 ; p<.001\right]$ and correctly classified $85.3 \%$ of $\mathrm{AD}$ patients and $76.5 \%$ of FTD patients.

To ensure that floor levels of performance were not attenuating profile differences between the FTD and AD groups, the analyses were repeated with only mildly-to-moderately (MDRS $\geq 105$ ) demented patients (FTD: $n=8$; AD: $n=$ 15). Although these subgroups did not differ significantly in age $[\mathrm{FTD}=61.0, \mathrm{AD}=65.9 ; t(21)=1.85 ; p=.08]$, education $[\mathrm{FTD}=14.2, \mathrm{AD}=14.1 ; t(21)<1]$, or total DRS scores $[\mathrm{FTD}=123.7, \mathrm{AD}=124.8 ; t(21)<1]$, profile analyses using RM MANOVA revealed a significant subscale $\times$ group interaction effect [multivariate $F(4,22)=$ 5.34; $p<.01]$ with FTD patients worse than AD patients on the Initiation/Perseveration and Conceptualization subscales, but better on the Attention, Construction, and Memory subscales (see Figure 2). The group differences on the Memory $[F(1,25)=7.49 ; p<.05 ; \mathrm{d}=1.03]$ and Conceptualization $[F(1,25)=6.36 ; p<.05 ; \mathrm{d}=1.12]$ subscales were significant.

A logistic regression model that included the Construction, Conceptualization, and Memory subscales was significant $\left[\chi^{2}(1,27)=23.24 ; p<.0005\right]$ and correctly classified $88.9 \%$ of AD and $88.9 \%$ of FTD patients in this mildly-tomoderately demented subgroup. The following logistic regression equation was obtained:

$$
\begin{aligned}
\beta^{*}= & -29.74+6.16(\text { Construction }) \\
& -0.87(\text { Conceptualization })+1.29 \text { (Memory) }
\end{aligned}
$$

where $1 /\left(1+e^{-\beta^{*}}\right)=$ probability of group membership at a cutoff of 0.33 (i.e., $<0.33=\mathrm{AD},>0.33=\mathrm{FTD}$ ). $\mathrm{A}$ cutoff of 0.33 was chosen to maximize FTD classification.

\section{Rates of Progression}

The RM ANOVA revealed a significant group $\times$ year interaction $[\mathrm{F}(1,34)=11.49 ; p<.01]$, reflecting differential rates of cognitive decline in FTD and AD as measured by total MDRS scores (see Figure 3). The ARC in total MDRS score was significantly different in the two groups and more than twice as large in the FTD patients as in the AD patients (see Table 2). The RM ANOVA with the individual MDRS subscales (see Figure 4) revealed significant group $\times$ year interaction effects on the Attention $[\mathrm{F}(1,34)=4.46 ; p<$ $.05]$, Conceptualization $[\mathrm{F}(1,34)=8.79 ; p<.05]$, and Memory $[\mathrm{F}(1,34)=6.45 ; p<.05]$ subscales with the ARC greater in FTD patients than AD patients on each of these subscales. There was a trend for a steeper decline on the Construction subscale for FTD patients than for AD patients $[\mathrm{F}(1,34)=3.11 ; p=.09]$, but this interaction did not reach statistical significance.

\section{DISCUSSION}

The results of the present study demonstrate that FTD and AD patients with similar levels of global dementia exhibit distinct profiles of cognitive impairment across the subscales of the MDRS. Performance on the Initiation/ Perseveration and Conceptualization subscales was worse in FTD than in AD patients, whereas performance on the Memory and Construction subscales was worse in AD than in FTD patients. This same general pattern was maintained when analyses were restricted to mildly-to-moderately demented FTD and AD patients, with the greatest discrepancy between these subgroups evident on the Conceptualization and Memory subscales. The distinct patterns of cognitive deficits were robust enough to correctly classify $82 \%$ of all FTD and AD patients, and $89 \%$ of mildly-tomoderately demented FTD and AD patients. These results are consistent with previous reports of distinct cognitive deficit profiles associated with FTD and AD on more comprehensive neuropsychological test batteries (Kertesz et al., 2003a; Mathuranath et al., 2000; Rascovsky et al., 2002;

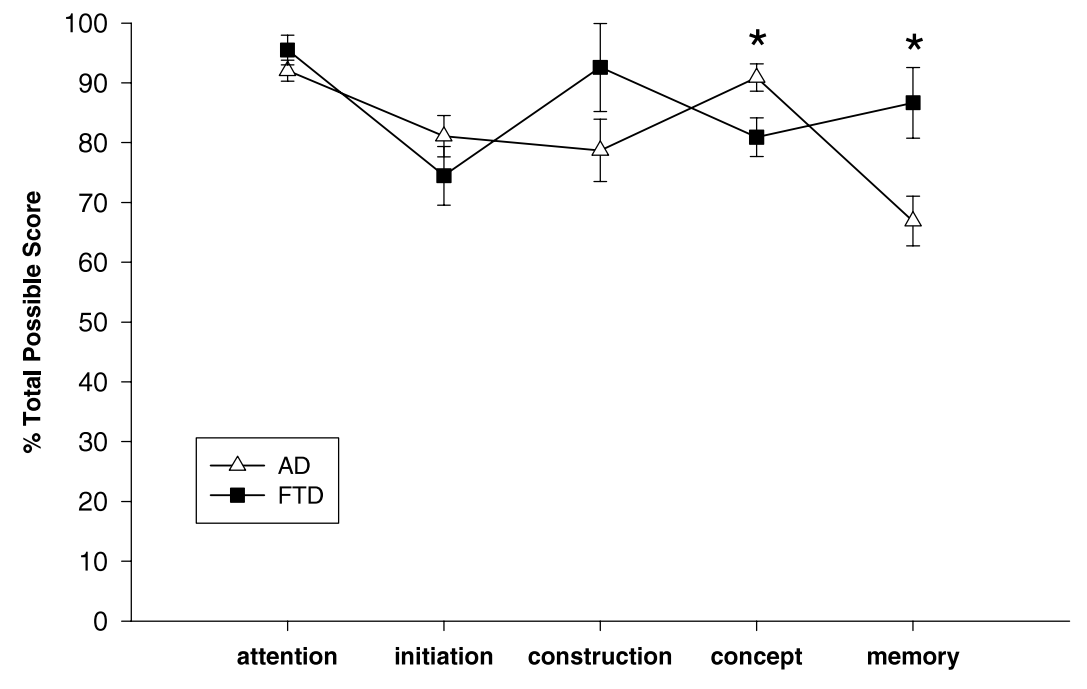

Fig. 2. Mean percentage (\%) of total possible score achieved by mildly-to-moderately demented (i.e., Mattis Dementia Rating Scale (MDRS) > 105) patients with frontotemporal dementia (FTD; squares) or Alzheimer's disease (AD; triangles) on the MDRS subscales. Error bars denote standard error of the mean. *Significant group difference, $p<.05$. 


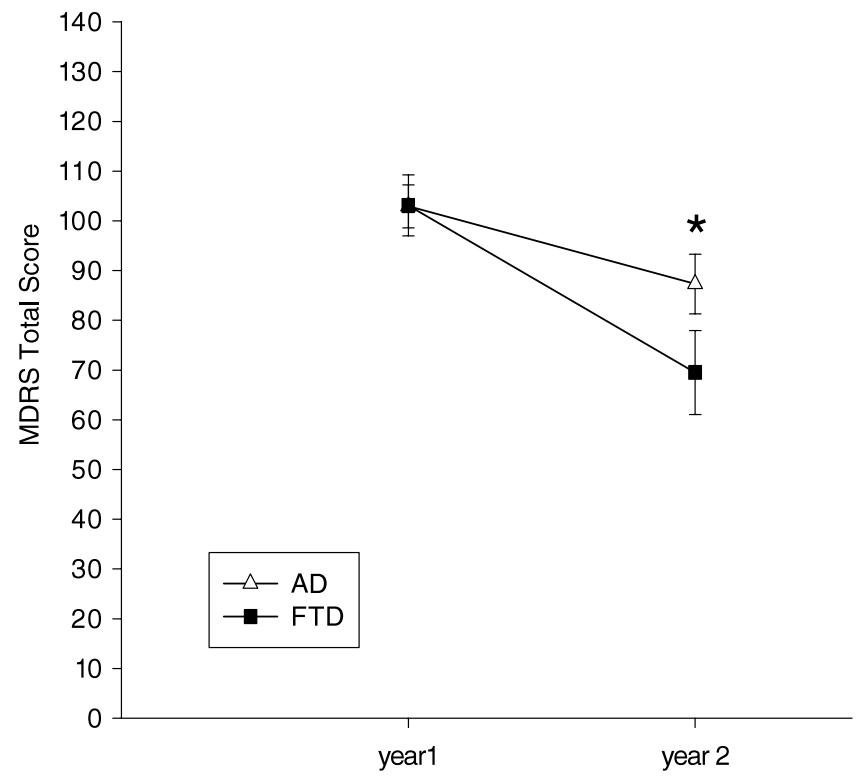

Fig. 3. Mean Mattis Dementia Rating Scale (MDRS) scores achieved by patients with frontotemporal dementia (FTD; squares) or Alzheimer's disease (AD; triangles) at baseline evaluation (year 1) and annual follow-up (year 2). Error bars denote SEM. $*$ Significant Group $\times$ Year interaction $(p<.05)$, indicating faster decline in FTD than AD patients.

Slachevsky et al., 2004) and extend these findings to show that the profiles are robust enough to be detected with the relatively brief MDRS.

The profiles of cognitive deficits exhibited by FTD and AD patients on the MDRS parallel those seen with more extensive testing. Several studies have shown greater memory impairment in AD than in FTD for both verbal (Elfgren et al., 1994; Kramer et al., 2003; Lindau et al., 1998; Perry \& Hodges, 2000; Rosen et al., 2004; Thomas-Anterion et al.,
2000) and visual (Frisoni et al., 1995; Kertesz et al., 2003a; Kramer et al., 2003; Pachana et al., 1996; Perry \& Hodges, 2000) materials, a finding that has been attributed to greater medial temporolimbic pathology in AD (Braak \& Braak, 1991) than in FTD (Frisoni et al., 1996; Lavenu et al., 1998). Similarly, a larger deficit in conceptual abilities in FTD than in AD has been shown on tests of conceptual processing and verbal abstraction (Kertesz et al., 2003a; Slachevsky et al., 2004). This particularly striking impairment in the conceptual abilities of FTD patients may reflect their more prominent frontal dysfunction compared with AD patients seen in both lesion and imaging studies (Lezak, 1995; Sarazin et al., 1998).

Although differential performances of FTD and AD patients on the Initiation/Perseveration and Construction MDRS subscales contributed to the distinct cognitive profiles they exhibited, group differences on these individual subscales only approached statistical significance. In the case of Initiation/Perseveration, this less than robust discriminability may be because the subscale is heavily weighted toward a semantically based verbal fluency task that could be adversely affected by either frontal lobe mediated executive processes (e.g., initiation, strategic search, and retrieval) prominently affected in FTD (Ruff et al., 1997) or by the deterioration of semantic knowledge that characterizes AD (Butters et al., 1987; Chertkow \& Bub, 1990; Martin \& Fedio, 1983; Monsch et al., 1994; Salmon et al., 1999). The relatively low discriminating power of the MDRS Construction subscale, in contrast, most likely reflects the cursory evaluation provided by this subscale. More rigorous tests have shown greater visuospatial and constructional deficits in AD than in FTD (Elfgren et al., 1994; Mendez et al., 1996; Miller et al., 1991; Rascovsky et al., 2002), and there are similar disparities in informant-based clinical reports of constructional abilities and topographical orientation (Varma et al., 1999). These differences are

Table 2. Mean annualized rate of change (ARC) on the Mattis Dementia Rating Scale total score and subscale scores for patients with frontotemporal dementia (FTD) or Alzheimer's disease (AD)

\begin{tabular}{lccr}
\hline \hline \multicolumn{1}{c}{ Mean ARC score } & FTD & AD & \\
& $N=12$ & $N=24$ & $p$ value \\
\hline Total score $(S D)$ & $-30.04(18.1)$ & $-14.82(10.7)$ & .003 \\
Range & $(0.0,-59.2)$ & $(5.3,-35.0)$ & \\
Attention (SD) & $-6.37(6.2)$ & $-3.05(4.0)$ & .062 \\
Range & $(0.0,-20.1)$ & $(2.0,-12.5)$ & .161 \\
Initiation/Perseveration $(S D)$ & $-6.89(4.4)$ & $-4.76(4.0)$ & .115 \\
Range & $(0.0,-12.4)$ & $(0.0,-14.5)$ & .008 \\
Construction $(S D)$ & $-1.20(1.2)$ & $-0.49(1.3)$ & \\
Range & $(0.0,-3.9)$ & $(1.8,-3.2)$ & .029 \\
Conceptualization $(S D)$ & $-10.51(9.6)$ & $(7.1,-13.8)$ & \\
Range & $(2.0,-24.7)$ & $-2.77(3.0)$ & $(1.1,-9.0)$ \\
Memory (SD) & $-5.07(2.5)$ & & \\
Range & $(-1.0,-9.0)$ & & \\
\hline \hline
\end{tabular}



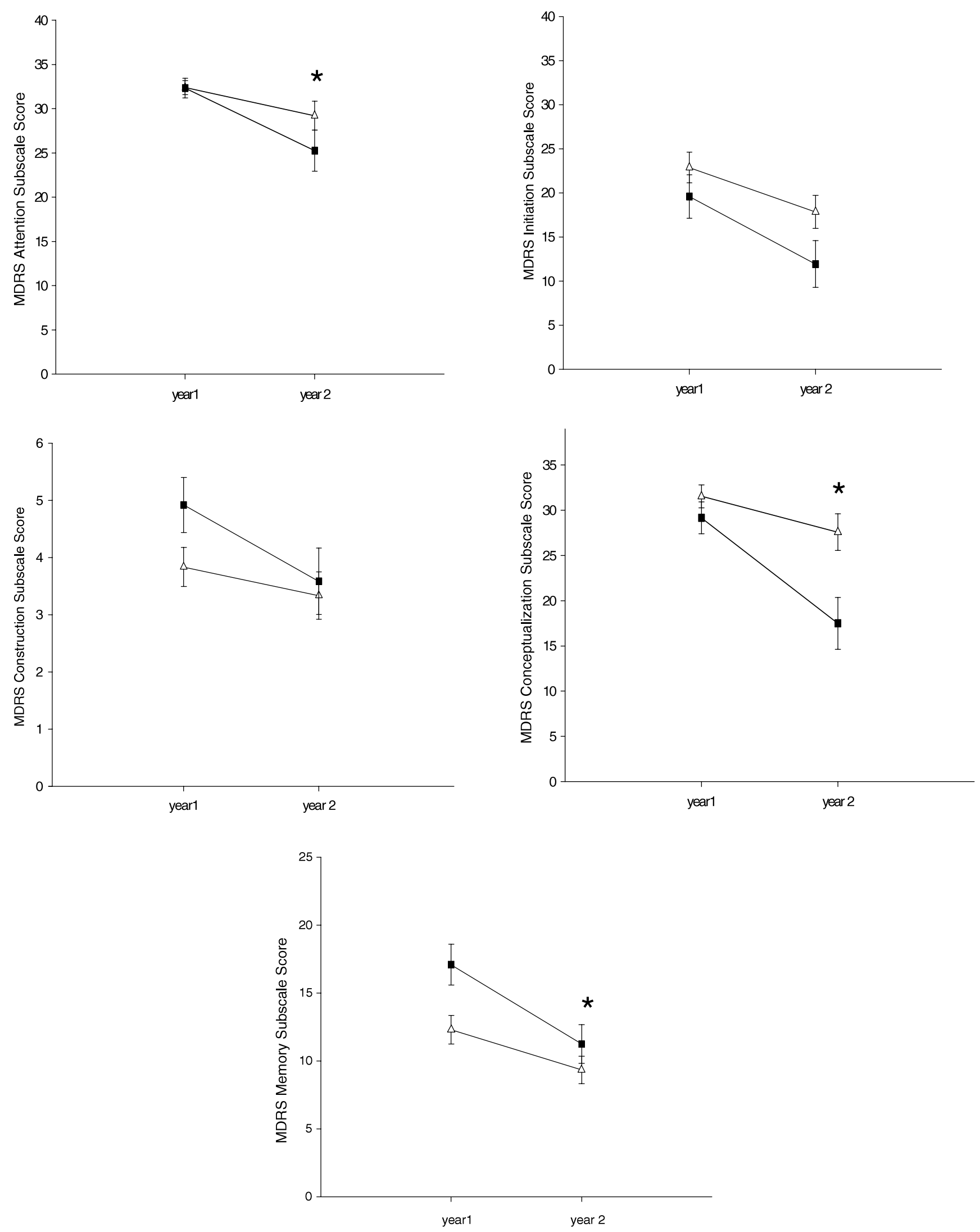

Fig. 4. Mean Attention, Initiation/Perseveration, Construction, Conceptualization, and Memory subscale scores achieved by patients with frontotemporal dementia (FTD; squares) or Alzheimer's disease (AD; triangles) at baseline evaluation (year 1) and annual follow-up (year 2). Error bars denote standard error of the mean. *Significant Group $\times$ Year interaction $(p<.05)$, indicating faster decline in FTD than AD patients. MDRS = Mattis Dementia Rating Scale. 
consistent with the relative sparing of occipitoparietal regions in FTD compared with AD. It should be noted that studies of visuospatial and constructional abilities that have not shown a difference between FTD and AD often involve drawing the Rey-Osterrieth Complex Figure (Diehl et al., 2005; Frisoni et al., 1995; Kertesz et al., 2003a; Kramer et al., 2003; Pachana et al., 1996; Rosen et al., 2004), a task known to be influenced by attentional and strategicorganizational requirements dependent upon the frontal lobes (Varma et al., 1999).

A second major finding from the present study is that FTD patients exhibit a faster rate of cognitive decline than those with AD when cognition is measured by the MDRS. Patients with FTD declined an average of 30 points on the MDRS over 1 year, double the average 15-point drop for AD patients. This observed disparity in cognitive decline parallels recent findings using clinically diagnosed (Binetti et al., 2000) and autopsy-confirmed (Rascovsky et al., 2005) patient samples. Indeed, a recent multicenter study of autopsy-confirmed patients (Rascovsky et al., 2005) found faster rates of cognitive decline in FTD than AD when cognition was assessed with the Mini-Mental State Examination (MMSE). Mildly to moderately demented FTD patients showed an annual decline of 6.7 points on the MMSE, whereas patients with $\mathrm{AD}$ declined only 2.3 points.

Unlike the MMSE, the MDRS allows examination of differential decline in the various cognitive abilities assessed by its subscales. The more rapid cognitive decline of FTD patients compared with $\mathrm{AD}$ patients was most evident on the MDRS Attention, Conceptualization, and Memory subscales. The rapid declines in attention and conceptualization are consistent with the progressive frontotemporal atrophy that characterizes FTD (Broe et al., 2003). It is somewhat surprising that performance on the Memory subscale declined more rapidly in FTD than in AD, but this may have occurred for two reasons. First, a higher percentage of AD patients (29\%) than FTD patients (8\%) were at or very near floor levels of performance on the Memory subscale at the initial evaluation (i.e., a score of 8 or below with 5 being the lowest possible score with chance performance), and this restricted range in measurement may have attenuated the group $\times$ year interaction effect. Second, it is likely that the FTD disease process eventually encroaches into medial temporal lobe structures that underlie memory performance (Broe et al., 2003). The failure to observe significant group differences in decline on the Construction subscale most likely reflects the cursory evaluation provided by this subscale. The relatively equivalent rate of decline for FTD and AD patients on the Initiation/ Perseveration subscale may occur because the semantic fluency task that dominates the subscale can be adversely influenced by decline in executive processes prominently affected by FTD or by semantic processes prominently affected by AD.

Several caveats should be considered when interpreting the present results. First, the MDRS may aid in differentiating between FTD and AD and in tracking their progres- sion, but a comprehensive neuropsychological test battery is likely to be more sensitive to the distinct cognitive profiles and rates of decline engendered by the two disorders (Rascovsky et al., 2002). There is little doubt, however, that the clinical contributions of the MDRS extends beyond simply evaluating the overall severity of cognitive loss in FTD and AD. Second, it is possible that the clinical and pathological heterogeneity within the FTD spectrum gives rise to cognitive profiles and rates of disease progression that differ by clinical or pathological subtype. Unfortunately, the relatively small sample size in the present study did not allow for such comparisons. Future studies using larger samples of autopsy-confirmed patients should be able to verify or refute this possibility. Third, given that the MDRS lacks a specific language subscale, it may not be ideal for detecting and tracking language-predominant presentations of FTD. Finally, it is possible that the FTD patient sample in the present study is not highly representative of the entire FTD population. As a specialized dementia center, the UCSD ADRC usually receives referrals for suspected cognitive impairment rather than the prominent behavioral dysfunction that may characterize early FTD. Despite these limitations, the present findings from autopsy-confirmed FTD and AD patients suggest that the MDRS might help to clinically distinguish between FTD and AD patients in the mildto-moderate stage of dementia, and may be more effective than other brief mental status examinations for tracking disease progression. Because of these characteristics, the MDRS might also prove to be useful for efficiently evaluating $\operatorname{cog}$ nitive treatment effects during clinical trials involving patients with FTD.

\section{ACKNOWLEDGMENTS}

This study was supported with funding from NIH grants P50 AG05131 and RO1 AG12963. We gratefully acknowledge the contribution of the late Dr. Leon J. Thal to the initial conceptualization of this study. The information in this manuscript and the manuscript itself has never been published either electronically or in print. The authors have no financial or other relationship that could constitute a conflict of interest.

\section{REFERENCES}

Aarsland, D., Litvan, I., Salmon, D.P., Galasko, D., WentzelLarsen, T., \& Larsen, J.P. (2003). Performance on the dementia rating scale in Parkinson's disease with dementia and dementia with Lewy bodies: Comparison with progressive supranuclear palsy and Alzheimer's disease. Journal of Neurology, Neurosurgery and Psychiatry, 74, 1215-1220.

Barker, W.W., Luis, C.A., Kashuba, A., Luis, M., Harwood, D.G., Loewenstein, D., Waters, C., Jimison, P., Shepherd, E., Sevush, S., Graff-Radford, N., Newland, D., Todd, M., Miller, B., Gold, M., Heilman, K., Doty, L., Goodman, I., Robinson, B., Pearl, G., Dickson, D., \& Duara, R. (2002). Relative frequencies of Alzheimer disease, Lewy body, vascular and frontotemporal dementia, and hippocampal sclerosis in the State of Florida Brain Bank. Alzheimer Disease and Associated Disorders, 16 , 203-212. 
Bier, J.C., Ventura, M., Donckels, V., Van Eyll, E., Claes, T., Slama, H., Fery, P., Vokaer, M., \& Pandolfo, M. (2004). Is the Addenbrooke's Cognitive Examination effective to detect frontotemporal dementia? Journal of Neurology, 251, 428-431.

Binetti, G., Locascio, J.J., Corkin, S., Vonsattel, J.P., \& Growdon, J.H. (2000). Differences between Pick disease and Alzheimer disease in the clinical appearance and rate of cognitive decline. Archives of Neurology, 57, 225-232.

Braak, H. \& Braak, E. (1991). Neuropathological staging of Alzheimer-related changes. Acta Neuropathologica, 82, 239-259.

Broe, M., Hodges, J.R., Schofield, E., Shepherd, C.E., Kril, J.J., \& Halliday, G.M. (2003). Staging disease severity in pathologically confirmed cases of frontotemporal dementia. Neurology, 60, 1005-1011.

Butters, N., Granholm, E., Salmon, D.P., Grant, I., \& Wolfe, J. (1987). Episodic and semantic memory: A comparison of amnesic and demented patients. Journal of Clinical and Experimental Neuropsychology, 9, 479-497.

Chertkow, H. \& Bub, D. (1990). Semantic memory loss in dementia of Alzheimer's type. What do various measures measure? Brain, 113, 397-417.

Coblentz, J.M., Mattis, S., Zingesser, L.H., Kasoff, S.S., Wisniewski, H.M., \& Katzman, R. (1973). Presenile dementia. Archives of Neurology, 29, 299-308.

Connor, D.J., Salmon, D.P., Sandy, T.J., Galasko, D., Hansen, L.A., \& Thal, L.J. (1998). Cognitive profiles of autopsy-confirmed Lewy body variant vs pure Alzheimer disease. Archives of Neurology, 55, 994-1000.

Diehl, J., Monsch, A.U., Aebi, C., Wagenpfeil, S., Krapp, S., Grimmer, T., Seeley, W., Forstl, H., \& Kurz, A. (2005). Frontotemporal dementia, semantic dementia, and Alzheimer's disease: The contribution of standard neuropsychological tests to differential diagnosis. Journal of Geriatric Psychiatry and Neurology, 18, 39-44.

Elfgren, C., Brun, A., Gustafson, L., Johanson, A., Minthon, L., Passant, U., \& Risberg, J. (1994). Neuropsychological tests as discriminators between dementia of Alzheimer's type and Frontotemporal dementia. International Journal of Geriatric Psychiatry, 9, 635-642.

Frisoni, G.B., Beltramello, A., Geroldi, C., Weiss, C., Bianchetti, A., \& Trabucchi, M. (1996). Brain atrophy in frontotemporal dementia. Journal of Neurology, Neurosurgery and Psychiatry, 61, 157-165.

Frisoni, G.B., Pizzolato, G., Geroldi, C., Rossato, A., Bianchetti, A., \& Trabucchi, M. (1995). Dementia of the frontal type: Neuropsychological and 99 TC-HMPAO SPECT features. Journal of Geriatric Psychiatry and Neurology, 8, 42-48.

Galasko, D.R., Gould, R.L., Abramson, I.S., \& Salmon, D.P. (2000). Measuring cognitive change in a cohort of patients with Alzheimer's disease. Statistics in Medicine, 19, 1421-1432.

Gardner, R., Jr., Oliver-Munoz, S., Fisher, L., \& Empting, L. (1981). Mattis Dementia Rating Scale: Internal reliability study using a diffusely impaired population. Journal of Clinical Neuropsychology, 3, 271-275.

Gislason, T.B., Sjogren, M., Larsson, L., \& Skoog, I. (2003). The prevalence of frontal variant frontotemporal dementia and the frontal lobe syndrome in a population based sample of 85 year olds. Journal of Neurology, Neurosurgery and Psychiatry, 74, 867-871.

Gould, R., Abramson, I., Galasko, D., \& Salmon, D.P. (2001). Rate of cognitive change in Alzheimer's disease: Methodolog- ical approaches using random effects models. Journal of the International Neuropsychological Society, 7, 813-824.

Grasbeck, A., Englund, E., Horstmann, V., Passant, U., \& Gustafson, L. (2003). Predictors of mortality in frontotemporal dementia: A retrospective study of the prognostic influence of prediagnostic features. International Journal of Geriatric Psychiatry, 18, 594-601.

Green, R.C., Woodard, J.L., \& Green, J. (1995). Validity of the Mattis Dementia Rating Scale for detection of cognitive impairment in the elderly. Journal of Neuropsychiatry and Clinical Neurosciences, 7, 357-360.

Gregory, C.A., Orrell, M., Sahakian, B., \& Hodges, J.R. (1997). Can frontotemporal dementia and Alzheimer's disease be differentiated using a brief battery of tests? International Journal of Geriatric Psychiatry, 12, 375-383.

Hansen, L.A. \& Samuel, W. (1997). Criteria for Alzheimer's disease and the nosology of dementia with Lewy bodies. Neurology, 48, 126-132.

Hodges, J.R., Davies, R., Xuereb, J., Kril, J., \& Halliday, G. (2003). Survival in frontotemporal dementia. Neurology, 61, 349-354.

Kertesz, A., Davidson, W., McCabe, P., \& Munoz, D. (2003a). Behavioral quantitation is more sensitive than cognitive testing in frontotemporal dementia. Alzheimer Disease and Associated Disorders, 17, 223-229.

Kertesz, A., Muñoz, D.G., \& Hillis, A. (2003b). Preferred terminology. Annals of Neurology, 54(Suppl. 5), S3-S6.

Khachaturian, Z.S. (1985). Diagnosis of Alzheimer's disease. Archives of Neurology, 42, 1097-1105.

Knopman, D.S., Mastri, A.R., Frey, W., Sung, J.H., \& Rustan, T. (1990). Dementia lacking distinctive histologic features: A common non-Alzheimer degenerative dementia. Neurology, 40, 251-256.

Kramer, J.H., Jurik, J., Sha, S.J., Rankin, K.P., Rosen, H.J., Johnson, J.K., \& Miller, B.L. (2003). Distinctive neuropsychological patterns in frontotemporal dementia, semantic dementia, and Alzheimer disease. Cognitive and Behavioral Neurology, 16, 211-218.

Lavenu, I., Pasquier, F., Lebert, F., Pruvo, J.P., \& Petit, H. (1998). Explicit memory in frontotemporal dementia: The role of medial temporal atrophy. Dementia and Geriatric Cognitive Disorders, 9, 99-102.

Lebert, F., Stekke, W., Hasenbroekx, C., \& Pasquier, F. (2004). Frontotemporal dementia: A randomised, controlled trial with trazodone. Dementia and Geriatric Cognitive Disorders, 17, 355-359.

Lezak, M.D. (1995). Neuropsychological Assessment. Oxford, UK: Oxford University Press.

Lindau, M., Almkvist, O., Johansson, S.E., \& Wahlund, L.O. (1998). Cognitive and behavioral differentiation of frontal lobe degeneration of the non-Alzheimer's type and Alzheimer's disease. Dementia and Geriatric Cognitive Disorders, 9, 205-213.

Litvan, I., Agid, Y., Sastrj, N., Jankovic, J., Wenning, G.K., Goetz, C.G., Verny, M., Brandel, J.P., Jellinger, K., Chaudhuri, K.R., McKee, A., Lai, E.C., Pearce, R.K., \& Bartko, J.J. (1997). What are the obstacles for an accurate clinical diagnosis of Pick's disease? A clinicopathologic study. Neurology, 49, 62-69.

Martin, A. \& Fedio, P. (1983). Word production and comprehension in Alzheimer's disease: The breakdown in semantic knowledge. Brain and Language, 19, 124-141.

Mathuranath, P.S., Nestor, P.J., Berrios, G.E., Rakowicz, W., \& 
Hodges, J.R. (2000). A brief cognitive test battery to differentiate Alzheimer's disease and frontotemporal dementia. Neurology, 55, 1613-1620.

Mattis, S. (1976). Mental status examination for organic mental syndrome in the elderly patient. In L. Bellak \& T.B. Karasu (Eds.), Geriatric Psychiatry: A Handbook for Psychiatrists and Primary Care Physicians (pp. 77-121). New York: Grune \& Stratton Inc.

Mattis, S. (1988). Dementia Rating Scale: Professional Manual. Odessa, FL: Psychological Assessment Resources.

McKhann, G.M., Albert, M.S., Grossman, M., Miller, B., Dickson, D., \& Trojanowski, J.Q. (2001). Clinical and pathological diagnosis of frontotemporal dementia: Report of the Work Group on Frontotemporal Dementia and Pick's Disease. Archives of Neurology, 58, 1803-1809.

Mendez, M.F., Cherrier, M., Perryman, K.M., Pachana, N., Miller, B.L., \& Cummings, J.L. (1996). Frontotemporal dementia versus Alzheimer's disease: Differential cognitive features. Neurology, 47, 1189-1194.

Mendez, M.F., Selwood, A., Mastri, A.R., \& Frey, W.H. (1993). Pick's disease versus Alzheimer's disease: A comparison of clinical characteristics. Neurology, 43, 289-292.

Miller, B.L., Cummings, J.L., Villanueva-Meyer, J., Boone, K., Mehringer, C.M., Lesser, I.M., \& Mena, I. (1991). Frontotemporal lobe degeneration: Clinical, neuropsychological and SPECT characteristics. Neurology, 41, 1374-1382.

Mirra, S.S., Heyman, A., \& Mohs, R.C. (1991). The Consortium to Establish a Registry for Alzheimer's disease (CERAD). Part II. Standardization of the neuropathologic assessment of Alzheimer's disease. Neurology, 41, 479-486.

Monsch, A.U., Bondi, M.W., Butters, N., Paulsen, J.S., Salmon, D.P., Brugger, P., \& Swenson, M. (1994). A comparison of category and letter fluency in Alzheimer's Disease and Huntington's Disease. Neuropsychology, 8, 25-30.

Monsch, A.U., Bondi, M.W., Salmon, D.P., Butters, N., Thal, L.J., Hansen, L.A., Weiderholt, W.C., Cahn, D.A., \& Klauber, M.R. (1995). Clinical validity of the Mattis Dementia Rating Scale in detecting Dementia of the Alzheimer type. A double crossvalidation and application to a community-dwelling sample. Archives of Neurology, 52, 899-904.

Moretti, R., Torre, P., Antonello, R.M., Cazzato, G., \& Bava, A. (2003). Frontotemporal dementia: Paroxetine as a possible treatment of behavior symptoms. A randomized, controlled, open 14-month study. European Neurology, 49, 13-19.

Neary, D. (1999). Overview of frontotemporal dementias and the consensus applied. Dementia and Geriatric Cognitive Disorders, 10(Suppl. 1), 6-9.

Neary, D., Snowden, J.S., Gustafson, L., Passant, U., Stuss, D., Black, S., Freedman, M., Kertesz, A., Robert, P.H., Albert, M., Boone, K., Miller, B.L., Cummings, J., \& Benson, D.F. (1998). Frontotemporal lobar degeneration: A consensus on clinical diagnostic criteria. Neurology, 51, 1546-1554.

Pachana, N.A., Boone, K.B., Miller, B.L., Cummings, J.L., \& Berman, N. (1996). Comparison of neuropsychological functioning in Alzheimer's disease and frontotemporal dementia. Journal of the International Neuropsychological Society, 2, 505-510.

Paolo, A.M., Troster, A.I., Glatt, S.L., Hubble, J.P., \& Koller, W.C. (1995). Differentiation of the dementias of Alzheimer's and Parkinson's disease with the Dementia Rating Scale. Journal of Geriatric Psychiatry and Neurology, 8, 184-188.

Pasquier, F., Fukui, T., Sarazin, M., Pijnenburg, Y., Diehl, J., Grundman, M., \& Miller, B.L. (2003). Laboratory investigations and treatment in frontotemporal dementia. Annals of Neurology, 54(Suppl. 5), S32-S35.

Pasquier, F., Richard, F., \& Lebert, F. (2004). Natural history of frontotemporal dementia: Comparison with Alzheimer's disease. Dementia and Geriatric Cognitive Disorders, 17, 253-257.

Paulsen, J.S., Butters, N., Sadek, J.R., Johnson, S.A., Salmon, D.P., Swerdlow, N.R., \& Swenson, M.R. (1995). Distinct cognitive profiles of cortical and subcortical dementia in advanced illness. Neurology, 45, 951-956.

Perry, R.J. \& Hodges, J.R. (2000). Differentiating frontal and temporal variant frontotemporal dementia from Alzheimer's disease. Neurology, 54, 2277-2284.

Pfeffer, R.J., Kurosaki, T.T., Harrah, C.H., Chance, J.M., \& Filos, S. (1982). Measurement of functional activities in older adults in the community. Journal of Gerontology, 37, 323-329.

Qume, M., Zeman, S., \& Stratmann, G.C. (1994). A neurochemical study of non-Alzheimer dementia. British Journal of Pharmacology, 94, 112.

Rahman, S., Robbins, T.W., \& Sahakian, B.J. (1999). Comparative cognitive neuropsychological studies of frontal lobe function: Implications for therapeutic strategies in frontal variant frontotemporal dementia. Dementia and Geriatric Cognitive Disorders, 10(Suppl.), 15-28.

Rascovsky, K., Salmon, D.P., Ho, G.J., Galasko, D., Peavy, G.M., Hansen, L.A., \& Thal, L.J. (2002). Cognitive profiles differ in autopsy-confirmed frontotemporal dementia and AD. Neurology, 58, 1801-1808.

Rascovsky, K., Salmon, D.P., Lipton, A.M., Leverenz, J.B., DeCarli, C., Jagust, W., Clark, C.M., Mendez, M.F., Tang-Wai, D.F., Graff-Radford, N.R., \& Galasko, D. (2005). Rate of progression differs in Frontotemporal Dementia and Alzheimer's Disease. Neurology, 65, 397-403.

Ratnavalli, E., Brayne, C., Dawson, K., \& Hodges, J.R. (2002). The prevalence of frontotemporal dementia. Neurology, 58, 1615-1621.

Rosen, H.J., Hartikainen, K.M., Jagust, W., Kramer, J.H., Reed, B.R., Cummings, J.L., Boone, K., Ellis, W., Miller, C., \& Miller, B.L. (2002). Utility of clinical criteria in differentiating frontotemporal lobar degeneration (FTLD) from AD. Neurology, $58,1608-1615$.

Rosen, H.J., Narvaez, J.M., Hallam, B., Kramer, J.H., Wyss-Coray, C., Gearhart, R., Johson, J.K., \& Miller, B.L. (2004). Neuropsychological and functional measures of severity in Alzheimer disease, frontotemporal dementia, and semantic dementia. Alzheimer Disease and Associated Disorders, 18, 202-207.

Rosser, A.E. \& Hodges, J.R. (1994). The Dementia Rating Scale in Alzheimer's disease, Huntington's disease and progressive supranuclear palsy. Journal of Neurology, 241, 531-536.

Rosso, S.M., Donker Kaat, L., Baks, T., Joosse, M., de Koning, I., Pijnenburg, Y., deJong, D., Dooijes, D., Kamphorst, W., Ravid, R., Niermeijer, M.F., Verheij, F., Kremer, H.P., \& Scheltens, P. (2003). Frontotemporal dementia in The Netherlands: Patient characteristics and prevalence estimates from a populationbased study. Brain, 126 (Pt 9), 2016-2022.

Ruff, R.M., Light, R.H., Parker, S.B., \& Levin, H.S. (1997). The psychological construct of word fluency. Brain and Language, 57, 394-405.

Salmon, D.P., Heindel, W.C., \& Lange, K. (1999). Differential decline in word generation from phonemic and semantic categories during the course of Alzheimer's disease: Implications for the integrity of semantic memory. Journal of the International Neuropsychological Society, 5, 692-703. 
Salmon, D.P., Kwo-on-Yuen, P.F., Heindel, W.C., Butters, N., \& Thal, L.J. (1989). Differentiation of Alzheimer's disease and Huntington's disease with the Dementia Rating Scale. Archives of Neurology, 46, 1204-1208.

Salmon, D.P., Thal, L.J., Butters, N., \& Heindel, W. (1990). Longitudinal evaluation of dementia of the Alzheimer type: A comparison of 3 standardized mental status examinations. Neurology, $40,1225-1230$.

Samuel, W., Galasko, D., Masliah, E., \& Hansen, L.A. (1996). Neocortical Lewy body counts correlate with dementia in the Lewy body variant of Alzheimer's disease. Journal of Neuropathology and Experimental Neurology, 55, 44-52.

Sarazin, M., Pillon, B., Giannakopoulos, P., Rancurel, G., Samson, Y., \& Dubois, B. (1998). Clinicometabolic dissociation of cognitive functions and social behavior in frontal lobe lesions. Neurology, 51, 142-148.

Shay, K.A., Duke, L.W., Conboy, T., Harrell, L.E., Callaway, R., \& Folks, D.G. (1991). The clinical validity of the Mattis Dementia Rating Scale in staging Alzheimer's dementia. Journal of Geriatric Psychiatry and Neurology, 4, 18-25.

Slachevsky, A., Villalpando, J.M., Sarazin, M., Hahn-Barma, V., Pillon, B., \& Dubois, B. (2004). Frontal assessment battery and differential diagnosis of frontotemporal dementia and Alzheimer disease. Archives of Neurology, 61, 1104-1107.

Smith, G.E., Ivnik, R.J., Malec, J.F., Kokmen, E., Tangalos, E., \& Petersen, R.C. (1994). Psychometric Properties of the Mattis Dementia Rating Scale. Assessment, 1, 123-132.

Sparks, R. \& Markesbery, W.R. (1991). Altered serotonergic and cholinergic synaptic markers in Pick's disease. Archives of Neurology, 48, 796-799.

Swartz, J.R., Miller, B.L., Lesser, I.M., \& Darby, A.L. (1997). Frontotemporal dementia: Treatment response to serotonin selective reuptake inhibitors. Journal of Clinical Psychiatry, $58,212-216$.

Thomas-Anterion, C., Jacquin, K., \& Laurent, B. (2000). Differential mechanisms of impairment of remote memory in Alzheimer's and frontotemporal dementia. Dementia and Geriatric Cognitive Disorders, 11, 100-106.

Troster, A.I., Moe, K.E., Vitiello, M.V., \& Prinz, P.N. (1994). Predicting long-term outcome in individuals at risk for Alzheimer's disease with the Dementia Rating Scale. The Journal of Neuropsychiatry and Clinical Neurosciences, 6, 54-57.

Varma, A.R., Snowden, J.S., Lloyd, J.J., Talbot, P.R., Mann, D.M., \& Neary, D. (1999). Evaluation of the NINCDS-ADRDA criteria in the differentiation of Alzheimer's disease and frontotemporal dementia. Journal of Neurology, Neurosurgery and Psychiatry, 66, 184-188.

Vitaliano, P.P., Breen, A.R., Russo, J., Albert, M., Vitiello, M.V., \& Prinz, P.N. (1984). The clinical utility of the dementia rating scale for assessing Alzheimer patients. Journal of Chronic Diseases, 37, 743-753.

Woodard, J.L., Salthouse, T.A., Godsall, R., \& Green, R.C. (1996). Confirmatory factor analysis of the Mattis Dementia Rating Scale in patients with Alzheimer's disease. Psychological Assessment, 8, 85-91. 